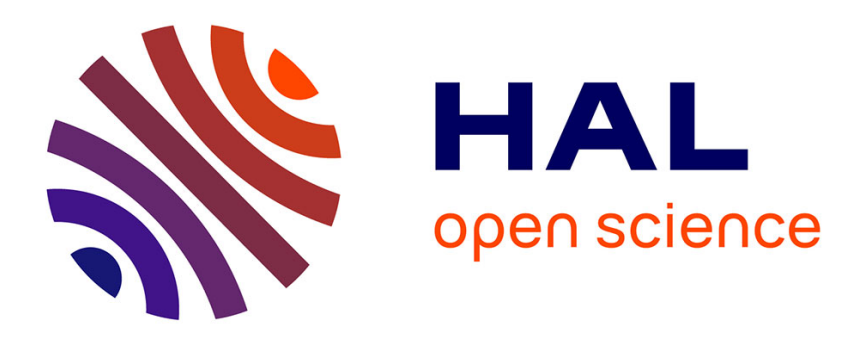

\title{
Random-energy model: An exactly solvable model of disordered systems
}

Bernard Derrida

\section{To cite this version:}

Bernard Derrida. Random-energy model : An exactly solvable model of disordered systems. Physical Review B, 1981, 24 (5), pp.2613-2626. 10.1103/PhysRevB.24.2613 . hal-03285956

\section{HAL Id: hal-03285956 https://hal.science/hal-03285956}

Submitted on 21 Jul 2021

HAL is a multi-disciplinary open access archive for the deposit and dissemination of scientific research documents, whether they are published or not. The documents may come from teaching and research institutions in France or abroad, or from public or private research centers.
L'archive ouverte pluridisciplinaire HAL, est destinée au dépôt et à la diffusion de documents scientifiques de niveau recherche, publiés ou non, émanant des établissements d'enseignement et de recherche français ou étrangers, des laboratoires publics ou privés. 


\title{
Random-energy model: An exactly solvable model of disordered systems
}

\author{
Bernard Derrida \\ Service de Physique Théorique, Centre d'Etudes Nucleaires de Saclay F-91190, Gif-sur-Yvette, France
}

(Received 2 February 1981)

\begin{abstract}
A simple model of disordered systems - the random-energy model-is introduced and solved. This model is the limit of a family of disordered models, when the correlations between the energy levels become negligible. The model exhibits a phase transition and the low-temperature phase is completely frozen. The corrections to the thermodynamic limit are discussed in detail. The magnetic properties are studied, and a constant susceptibility is found at low temperature. The phase diagram in the presence of ferromagnetic pair interactions is described. Many results are qualitatively the same as those of the Sherrington-Kirkpatrick model. The problem of using the replica method is analyzed. Lastly, this random-energy model provides lower bounds for the ground-state energy of a large class of spin-glass models.
\end{abstract}

\section{INTRODUCTION}

Recently, many authors have studied the Sherrington-Kirkpatrick model ${ }^{1}$ (the SK model). The first interest of the SK model was to try to understand what kind of mean-field theory ${ }^{2}$ should be valid for spin-glass models. ${ }^{3}$ The second important interest was to know why the replica method used by $\mathrm{SK}^{1}$ was incorrect. The SK model is simple enough to allow the calculation of all the integer moments $\left\langle Z^{n}\right\rangle$ of the partition function in the thermodynamic limit. Because of the too rapid growth of these moments $\left\langle Z^{n}\right\rangle$ (see Appendix A), the continuation to noninteger values of $n$ is not unique. ${ }^{4}$ In order to justify why the continuation $n \rightarrow 0$ used by SK did not provide $\langle\ln Z\rangle$, a breaking of symmetry in the replica space was proposed. ${ }^{5}$ Several works ${ }^{6,7}$ followed this idea and tried to investigate whether calculations with a broken symmetry in the replica space could lead to the true expression of $\langle\ln Z\rangle$. At present the situation is not yet clear and no simple analytic solution of the SK model has yet been derived. Therefore, it is interesting to study the random-energy model ${ }^{8}$ which is simpler but contains most of the difficulties encountered in the SK model.

The random-energy model (RE) describes a system whose energy levels are independent random variables. The model can be defined without specifying any microscopic Hamiltonian. However one can find a family of spin-glass models which generalize the SK model and give in a certain limit the RE model. Because of its simplicity, the moments $\left\langle Z^{n}\right\rangle$ and the average free energy $\langle\ln Z\rangle$ are given by very simple expressions. The first motivation to study this RE model was that the behavior of the $\left\langle Z^{n}\right\rangle$ is the same as in the SK model and that the impossibility of using replicas is even more evident here. Another motivation was to look at the approach to the thermo- dynamic limit. Thouless, Anderson, and Palmer ${ }^{2}$ found that the transition temperature of the SK model could be seen in the $1 / N$ correction of the free energy. Moreover the approach to the thermodynamic limit is always a difficulty of numerical simulations. So it was interesting to compare the finite-size corrections of the RE model with those which are conjectured for the SK model. The main result is that the system exhibits a phase transition and that in the low-temperature phase the system is completely frozen. Another conclusion is that the infinite temperature is an accumulation point of critical temperatures where the corrections to the thermodynamic limit change their behavior. So one has to be very careful in the high-temperature expansion for these random systems. The definition of the RE model can be extended to cases where a magnetic field is present or where there are ferromagnetic pair interactions in addition to the random interactions. The phase diagrams are qualitatively the same as in the SK model. Finally, the RE model leads me to consider an approximation to spin-glass models in any dimension and gives lower bounds for the ground-state energies.

The main purpose of this work is to present in a detailed way the derivation of the results announced in a previous Letter. ${ }^{8}$ The paper is organized as follows: in Sec. II, the SK model is generalized and the RE model is obtained as a limit of a family of spinglass models. In Sec. III the definition and a simple solution of the RE model are given. These two sections are just a recall of the arguments contained in Ref. 8. In Sec. IV, the moments $\left\langle Z^{n}\right\rangle$ are calculated and the problem of finding $\langle\ln Z\rangle$ using these moments is discussed. In Sec. V, the approach to the thermodynamic limit is studied. The replica method used for the RE model is described in Sec. VI and surprisingly an unstable saddle point gives the low- 
temperature free energy. In Secs. VII and VIII, the behavior of the RE model in a magnetic field and in the presence of ferromagnetic pair interactions is studied. In Sec. IX, a lower bound of the ground-state energy of some spin-glass models in finite dimensions is obtained. Lastly in Sec. X, an attempt of expanding spin-glass models around the RE model is presented.

\section{RELATED MODELS}

In this section, I consider a family of models which generalize the SK model and I explain how the RE model is related to these models. The SK model is defined by the Hamiltonian

$$
\mathfrak{H}=-\sum_{\langle i j\rangle} J_{i j} \sigma_{i} \sigma_{j}
$$

where the spins $\sigma_{i}$ are Ising spins and where the $J_{i j}$ are random-quenched variables with probability distribution

$$
\rho\left(J_{i j}\right)=\left(\frac{N}{2 \pi J^{2}}\right)^{1 / 2} \exp \left(-\frac{J_{i j}^{2} N}{2 J^{2}}\right) .
$$

The model is infinite ranged because there is an interaction $J_{i j}$ for any pair of spins in the system.

One can generalize the SK model by replacing the random pair interactions in Eq. (1) by random $p$-spin interactions. The Hamiltonian is then

$$
\boldsymbol{\mathcal { C }}_{p}=-\sum_{i_{1}, \ldots, i_{p}} A_{i_{1}}, \ldots, i_{p} \sigma_{i_{1}}, \ldots, \sigma_{i_{p}} .
$$

Here again, the spins are Ising spins and there is an interaction $A_{i_{1}}, \ldots, i_{p}$ for any group of $p$ spins in the model. As in the SK model, the probability distribution of $A_{i_{1}}, \ldots, i_{p}$ has to be scaled with $N$ in order to ensure an extensive free energy

$$
\begin{aligned}
& \rho\left(A_{i_{1}}, \ldots, i_{p}\right) \\
& \quad=\left(\frac{N^{p-1}}{\pi J^{2} p !}\right)^{1 / 2} \exp \left(-\frac{\left(A_{i_{1}}, \ldots, i_{p}\right)^{2} N^{p-1}}{J^{2} p !}\right) .
\end{aligned}
$$

The relation between all these models can be seen in the one-level probability distribution $P(E)$. For a given configuration of the spins $\left\{\sigma_{i}^{(1)}\right\}$, the energy $E$ depends on the interactions $A_{i_{1}} \ldots, i_{p}$ of the Hamiltonian $\mathcal{K}_{p}$. By definition $P(E)$ is the probability that this configuration has energy $E$. Using Eqs. (3) and (4), one finds that

$$
P(E) \sim \exp \left(-\frac{E^{2}}{N J^{2}}\right) .
$$

This result justifies a posteriori the distribution chosen in Eq. (4) because all the Hamiltonians $\mathfrak{K}_{p}$ give the same $P(E)$. One can notice that $P(E)$ does not depend on the configuration of spins $\left\{\sigma_{i}^{(1)}\right\}$. One can also define the probability distribution $P_{1,2}\left(E_{1}, E_{2}\right)$ as the probability that two given configurations of spins $\left\{\sigma_{i}^{(1)}\right\}$ and $\left\{\sigma_{i}^{(2)}\right\}$ have, respectively, energies $E_{1}$ and $E_{2}$. It turns out that this probability distribution depends only on the distance between the two configurations, namely, on the number $N x$ of identical spins in the two configurations. One finds

$$
\begin{aligned}
P_{1,2}\left(E_{1}, E_{2}\right) \sim \exp & -\frac{\left(E_{1}+E_{2}\right)^{2}}{2 N J^{2}\left[1+(2 x-1)^{p}\right]} \\
& \left.-\frac{\left(E_{1}-E_{2}\right)^{2}}{2 N J^{2}\left[1-(2 x-1)^{p}\right]}\right) .
\end{aligned}
$$

The parameter $x$ is always between 0 and 1 . Therefore, when $p$ becomes large, one gets ${ }^{8}$

$$
P_{1,2}\left(E_{1}, E_{2}\right) \sim P\left(E_{1}\right) P\left(E_{2}\right) .
$$

One can consider also the probability distributions of three or more levels and again when $p$ is large these probability distributions become factorized. So when $p$ is large, the energy levels become independent random variables. The large- $p$ limit must always be taken after the thermodynamic limit $N \rightarrow \infty$ and so $p$ is much smaller than $N$. The RE model is defined as a system of $2^{N}$ independent random energy levels distributed according to $P(E)$ given by Eq. (5). One can notice that the SK model which corresponds to $p=2$ lies between the case $p=1$ which is a model of free spins in a random magnetic field and $p=\infty$ which is the RE model. So some of its properties should be well approximated by an interpolation between these two exactly solvable models.

\section{DEFINITION OF THE RANDOM-ENERGY MODEL}

The random-energy model is defined by the following three properties.

(i) The system has $2^{N}$ energy levels $E_{i}$.

(ii) These energy levels are distributed according to the probability distribution

$$
P(E)=\left(N \pi J^{2}\right)^{-1 / 2} \exp \left(-E^{2} / N J^{2}\right) .
$$

(iii) The energy levels $E_{i}$ are independent random variables.

The first two properties are actual features of some spin-glass models like the models defined by the Hamiltonians $\mathfrak{K}_{p}$ in Eq. (3). The third property is specific to this model. It simplifies the model enough to allow us to solve it exactly. However it can be seen as a crude approximation to more realistic models since the correlations between the energy levels are ignored.

One system is given by the choice of the $2^{N}$ energy 
levels $E_{i}$. For each system the partition function $Z$ is

$$
Z\left(\left\{E_{i}\right\}\right)=\sum_{i=1}^{2^{N}} \exp \left(-\frac{E_{i}}{T}\right)
$$

and as usual for disordered systems, one wants to calculate the average free energy.

$$
\begin{aligned}
F & =-T\langle\ln Z\rangle \\
& =-T \int \prod_{i}\left[P\left(E_{i}\right) d E_{i}\right] \ln Z\left(\left\{E_{i}\right\}\right) .
\end{aligned}
$$

The calculation of this average is done in the Appendix B and the results are discussed in Sec. V. Here I just recall the simple argument of Ref. 8 because it will be useful in Secs. VII and VIII.

For one sample of the $2^{N}$ energy levels, one can define $n(E)$ the number of energy levels belonging to the interval $(E, E+d E)$. This number fluctuates from one sample to another. It can be calculated by

$$
n(E)=\sum_{i=1}^{2^{N}} y_{i}
$$

where $y_{i}=1$ if $E<E_{i}<E+d E$ and $y_{i}=0$ otherwise. The average $\langle n(E)\rangle$ is easily obtained from the probability distribution of the energies $E_{i}$ :

$$
\langle n(E)\rangle=2^{N}\left\langle y_{i}\right\rangle=2^{N} \exp \left(-\frac{E^{2}}{N J^{2}}\right) A d E
$$

with $A=\left(N \pi J^{2}\right)^{-1 / 2}$. In order to have a well-defined energy in the thermodynamic limit $d E$ has to be small enough ( $d E \sim N^{\alpha}$ with $\left.\alpha<1\right)$. On the other hand, if one wants $n(E)$ to be rather smooth, $d E$ must be large enough. It turns out that one can choose $d E \sim N^{\alpha}$ for any $\alpha<1$. Then from Eq. (12) it is clear that there exists a critical energy $E_{0}$ defined by

$$
E_{0} / N=J(\ln 2)^{1 / 2} .
$$

If $|E|<E_{0}$, the average number $\langle n(E)\rangle$ of levels is much larger than 1. Because of the statistical independence of the energy levels, the fluctuations of $n(E)$ around its average are of order $\langle n(E)\rangle^{1 / 2}$ and are small compared with $\langle n(E)\rangle$. Therefore

$$
n(E) \sim\langle n(E)\rangle .
$$

If $|E|>E_{0}$, the average number $\langle n(E)\rangle$ is much smaller than 1 . So for almost all the systems $n(E)=0$ and with a very small probability which vanishes exponentially with $N, n(E) \geqslant 1$.

It is then clear that in the thermodynamic limit, if $|E|<E_{0}$, the entropy $S(E)$ is given by

$$
S(E)=N\left[\ln 2-\left(\frac{E}{N J}\right)^{2}\right]
$$

and there is no energy level larger than $E_{0}$ in absolute value. From the function $S(E)$, one can calcu- late the average free energy $F$ using the relation $d S / d E=1 / T$. One finds ${ }^{8}$

$$
N^{-1} F=\left\{\begin{array}{l}
-T \ln 2-J^{2} / 4 T, \quad \text { if } T>T_{c}, \\
-E_{0} / N=-J(\ln 2)^{1 / 2}, \quad \text { if } T<T_{c},
\end{array}\right.
$$

where

$$
T_{c}=J / 2(\ln 2)^{1 / 2} .
$$

Equation (16) follows directly from Eq. (15). When the temperature decreases until $T_{c}$, the energy $E$ reaches $-E_{0}$. For lower temperature than $T_{c}$, the energy of the system remains $E_{0}$ because there is no level of lower energy.

The fact that the average free energy could be obtained by calculating the average entropy is not surprising. The entropy and the free energy are related by a linear relation and so their averages are related by the same linear relation.

\section{MOMENTS}

The usual approach of disordered problems by replicas starts by calculating the moments $\left\langle Z^{n}\right\rangle$ of the partition function. Because of the statistical independence of the energies in the RE model, the expression of the moments $\left\langle Z^{n}\right\rangle$ is particularly simple. For the first three moments, one finds

$$
\begin{aligned}
\langle Z\rangle= & 2^{N}\left\langle e^{-E / T}\right\rangle=2^{N} e^{N \lambda^{2} / 4} \\
\left\langle Z^{2}\right\rangle= & 2^{N} e^{N \lambda^{2}}+2^{N}\left(2^{N}-1\right) e^{N \lambda^{2} / 2} \\
\left\langle Z^{3}\right\rangle= & 2^{N} e^{9 N \lambda^{2} / 4}+3\left[2^{N}\left(2^{N}-1\right)\right] e^{5 N \lambda^{2} / 4} \\
& +2^{N}\left(2^{N}-1\right)\left(2^{N}-2\right) e^{3 N \lambda^{2} / 4}
\end{aligned}
$$

where $\lambda^{2}=J^{2} / T^{2}$

To calculate any moment $\left\langle Z^{n}\right\rangle$, one can develop the $n$th power of $Z$ and then average

$$
\begin{aligned}
Z^{n}= & \sum_{\left\{p_{i}\right\}} \alpha\left(p_{1}, p_{2}, \ldots, p_{2^{N}}\right) \\
& \quad \times \exp \left(-\frac{p_{1} E_{1}}{T}-\frac{p_{2} E_{2}}{T}-\ldots,-\frac{p_{2^{N}} E_{2^{N}}}{T}\right),
\end{aligned}
$$

where $\alpha\left(p_{1}, p_{2}, \ldots\right)$ is a combinatorial factor

$$
\alpha\left(p_{1}, p_{2}, \ldots\right)=\frac{n !}{\prod_{i}\left(p_{i} !\right)}
$$

and where the sum is performed over all the integer partitions of $n$ :

$$
p_{i} \geqslant 0, \quad \sum_{i} p_{i}=n
$$

After the average, one can group the terms which 
have the same $\left\{p_{i}\right\}$ through a permutation of index. Each integer partition of $n$ is characterized by the numbers $\nu_{p}$ of $p_{i}$ which are equal to $p$. One finds at the end

$$
\begin{aligned}
\left\langle Z^{n}\right\rangle=\sum_{|\nu|} & \frac{\left(2^{N}\right) !}{\prod_{p=0}^{n}\left(\nu_{p}\right) !} \frac{n !}{\prod_{p=0}^{n}(p !)^{\nu} p} \\
& \times \exp \left(N \sum_{p=0}^{n} p^{2} \frac{\lambda^{2}}{4} v_{p}\right),
\end{aligned}
$$

where the sum has to be done over all the different choices of the integers $\nu_{p}$ which verify:

$$
\begin{aligned}
& \nu_{p} \geqslant 0, \\
& \sum_{p=0}^{n} v_{p}=2^{N}, \quad \sum_{p=1}^{n} p v_{p}=n .
\end{aligned}
$$

From the exact expression (20), one can derive the asymptotic behavior $(N \rightarrow \infty)$ of all these moments. First it is easy to replace each term in the sum (20) by its asymptotic behavior. Because of Eq. (21), all the $\nu_{p}$ for $p \geqslant 1$ are small and $\nu_{0}$ is of order $2^{N}$. One finds that

$$
\frac{\left(2^{N}\right) !}{\nu_{0} !} \sim 2^{N \Sigma_{p=1}^{n} v_{p}}
$$

and so

$$
\begin{aligned}
\left\langle Z^{n}\right\rangle \sim \sum_{[v]} & \frac{n !}{\prod_{p=1}^{n}\left(\nu_{p}\right) ! \prod_{p=1}^{n}(p !)^{\nu}} \\
& \times \exp N\left[\sum_{p=1}^{n} v_{p}\left(\ln 2+p^{2} \frac{\lambda^{2}}{4}\right)\right] .
\end{aligned}
$$

Now we can see what is the dominant term in the sum (22). Because of the constraint (21), one finds that (i) if $n \lambda^{2}>4 \ln 2$, the set of $\nu_{p}$ which gives the dominant contribution is $\nu_{n}=1$ and $\nu_{p}=0$ for $1 \leqslant p \leqslant n-1$; and (ii) if $n \lambda^{2}<4 \ln 2$, the best choice is $\nu_{1}=n$ and $\nu_{p}=0$ for $p \geqslant 2$. Therefore for each moment $\left\langle Z^{n}\right\rangle$ there is a critical temperature

$$
T_{n}=\sqrt{n} \frac{J}{2 \sqrt{\ln 2}}=\sqrt{n} T_{c}
$$

and the asymptotic behavior of $\left\langle Z^{n}\right\rangle$ is given by

$\left\langle Z^{n}\right\rangle \sim\left\{\begin{array}{l}\exp N n\left(\ln 2+\frac{J^{2}}{4 T^{2}}\right)=\langle Z\rangle^{n}, \quad \text { if } T>T_{n}, \\ \exp N\left(\ln 2+n^{2} \frac{J^{2}}{4 T^{2}}\right), \quad \text { if } T<T_{n} .\end{array}\right.$

At this stage, it is interesting to make a few remarks.

First, all the integer moments $\left\langle Z^{n}\right\rangle$ for $n \geqslant 2$ have a transition temperature $T_{n}$ (see Fig. 1). For the SK

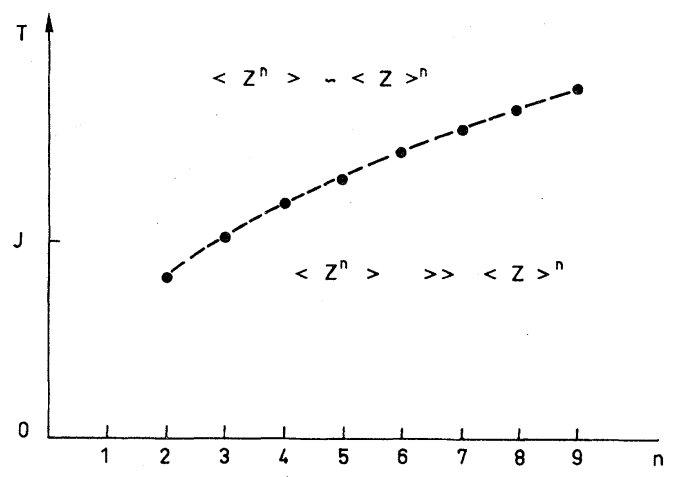

FIG. 1. The critical temperatures $T_{n}=\sqrt{n} T_{c}$ of the moments $\left\langle Z^{n}\right\rangle$ of the partition function. In the hightemperature region $T>T_{n},\left\langle Z^{n}\right\rangle \sim\langle Z\rangle^{n}$. In the lowtemperature region $T\left\langle T_{n},\left\langle Z^{n}\right\rangle\right.$ is much larger than $\langle Z\rangle^{n}$.

model the $\left\langle Z^{n}\right\rangle$ have also a transition temperature. ${ }^{9}$ The behavior of these moments are the same in the RE model and in the SK model. For the SK model, Eq. (24) is true whereas Eq. (23) is only valid for large $n$ and Eq. (25) is valid at very low temperature.

The simplest way to compute the average free energy would be to assume that the partition function $Z$ has small fluctuations around its average $\langle Z\rangle$. At infinite temperature, there is no fluctuation at all $\left(Z=2^{N}\right)$ and it seems reasonable that at high temperature, the probability distribution of $Z$ is concentrated around $\langle Z\rangle$. Then $\langle\ln Z\rangle$ could be obtained by

$$
\begin{aligned}
\langle\ln Z\rangle & =\ln \langle Z\rangle+\left\langle\ln \left(1+\frac{Z}{\langle Z\rangle}-1\right)\right\rangle \\
& =\ln \langle Z\rangle+\sum_{n=2}^{\infty} \frac{(-)^{n+1}}{n}\left\langle\left(\frac{Z}{\langle Z\rangle}-1\right)^{n}\right\rangle .
\end{aligned}
$$

From Eq. (25), it is clear that even at very high temperature, if $n$ is large enough $\left(n>4 T^{2} \ln 2 / J^{2}\right),\left\langle Z^{n}\right\rangle$ is given by its low-temperature expression and one has

$$
\left.\left\langle Z^{n}\right\rangle>\right\rangle\langle Z\rangle^{n}
$$

This proves that Eq. (26) is a divergent series at any temperature except $T=\infty$ and cannot be used to calculate $\langle\ln Z\rangle$.

Expressions (24) and (25) give the behavior of all the moments $\left\langle Z^{n}\right\rangle$. At any finite temperature, these moments for large $n$ behave like the exponential of $n^{2}$. It is known that when the growth of the moments is too rapid, there are many distributions which have the same moments. ${ }^{10}$ An example where the moments increase in the same way as the moments $\left\langle Z^{n}\right\rangle$ is given in the Appendix A. This example shows that the exact knowledge of the moments 
is not sufficient in this case to calculate $\langle\ln Z\rangle$.

In the microcanonical ensemble, the quantity of interest is the density $n(E)$ defined by Eq. (11). The moments of $n(E)$ can be calculated as those of $Z$. One develops the $q$ th power of Eq. (11) and one averages using the fact that

$$
\left\langle\left(y_{i}\right)^{p_{i}}\right\rangle=\left\{\begin{array}{l}
A \exp \left(-\frac{E^{2}}{N J^{2}}\right) d E=\langle y\rangle, \quad p_{i} \geqslant 1, \\
1, \quad p_{i}=0 .
\end{array}\right.
$$

The result is

$$
\left\langle[n(E)]^{q}\right\rangle=\sum_{\{v\rangle} \frac{\left(2^{N}\right) !}{\prod_{p=0}^{q}\left(\nu_{p}\right) !} \frac{q !}{\prod_{p=0}^{q}(p !)^{\nu_{p}}}(\langle y\rangle)^{\Sigma_{p}^{q}-1 \nu_{p}},
$$

where the integers $v_{p}$ verify Eq. (21). In the thermodynamic limit, Eq. (27) becomes simpler:

$$
\left\langle[n(E)]^{q}\right\rangle \sim \sum_{|v|} \frac{q !}{\prod_{p=1}^{q}\left(\nu_{p}\right) ! \prod_{p=1}^{q}(p !)^{\nu_{p}}}\langle n(E)\rangle^{\Sigma_{p}^{q}-1^{\nu_{p}}} .
$$

By looking at the dominant term in Eq. (28), one finds that the energy $E_{0}=J \sqrt{\ln 2}$ is a critical energy for all these moments.

If $|E|>E_{0}$, the dominant contribution comes from $v_{q}=1$ and $v_{p}=0$ (for $1 \leqslant p \leqslant q-1$ ), therefore

$$
\left\langle[n(E)]^{q}\right\rangle \sim\langle n(E)\rangle
$$

if $|E|<E_{0}$, the choice is $\nu_{1}=q$ and $\nu_{p}=0$ (for $p \geqslant 2$ ) and so

$$
\left\langle[n(E)]^{q}\right\rangle \sim\langle n(E)\rangle^{q} .
$$

So, for the RE model, the moments of the density $n(E)$ have a simpler behavior than those of $Z$. The fact that all the moments of $n(E)$ have the same critical energy indicates that this energy is the critical energy of the quenched system. The growth of the moments $\left\langle[n(E)]^{q}\right\rangle$ is at most an exponential of $q$ because the maximum value of $n(E)$ is always less than $2^{N}$. So there is in principle no problem about the unicity of the distribution of $n(E)$, once we know its moments.

\section{FINITE-SIZE CORRECTIONS}

The direct calculation of the average free energy is given in the Appendix B. The purpose of this section is only to discuss the results.

The first result is that there is a transition at temperature $T_{c}=J / 2 \sqrt{\ln 2}$. Of course, one recovers by averaging $\ln Z$, the extensive part of the free energy given in Eqs. (16) and (17).
At low temperature, the average free energy is

$$
\begin{aligned}
\frac{1}{N}\langle F\rangle= & -J \sqrt{\ln 2}+\frac{T_{c}}{2} \frac{\ln N}{N}+\frac{T_{c}}{2 N} \ln (4 \pi \ln 2) \\
& +\left(T_{c}-T\right) \frac{1}{N} \Gamma^{\prime}(1)-\frac{T_{c}}{N} \ln \left[\Gamma\left(1-\frac{T}{T_{c}}\right)\right] \\
& \left.+O\left(\frac{\ln N}{N}\right)^{2}\right)
\end{aligned}
$$

In the thermodynamic limit, the specific heat $C$ per spin vanishes below $T_{c}$ (Fig. 2). This means that the system is completely frozen in the whole low-temperature phase. From Eq. (31), one can compute the $1 / N$ corrections to $C$ :

$\left.C=\frac{1}{N} \frac{T}{T_{c}}\left[\frac{\Gamma^{\prime \prime}\left(1-\frac{T}{T_{c}}\right)}{\Gamma\left(1-\frac{T}{T_{c}}\right)}-\left(\frac{\Gamma^{\prime}\left(1-\frac{T}{T_{c}}\right)}{\Gamma\left(1-\frac{T}{T_{c}}\right)}\right)\right]^{2}\right]$.

This shows that numerical studies which are always done on finite systems would encounter difficulties in predicting a zero specific heat because of the slow convergence $(1 / N)$ to the thermodynamic limit. In the low-temperature phase, one finds also that the $1 / N$ correction is singular at $T_{c}$ because of the presence of $\ln \left[\Gamma\left(1-T / T_{c}\right)\right]$ in the expression (31).

The average ground-state energy $E_{\mathrm{GS}}$ is

$$
\begin{aligned}
-\frac{1}{N}\left\langle E_{\mathrm{GS}}\right\rangle= & +J \sqrt{\ln 2}-\frac{T_{c}}{2} \frac{\ln N}{N}-\frac{T_{c}}{2 N} \ln (4 \pi \ln 2) \\
& -\frac{T_{c}}{N} \Gamma^{\prime}(1)+O\left(\frac{1}{N}\right)
\end{aligned}
$$

The corrections are of order $N^{-1} \ln N$ and are comparable to the $N^{-1}$ (Ref. 1) and the $N^{-1 / 2}$ (Ref. 11) corrections conjectured for the SK model.

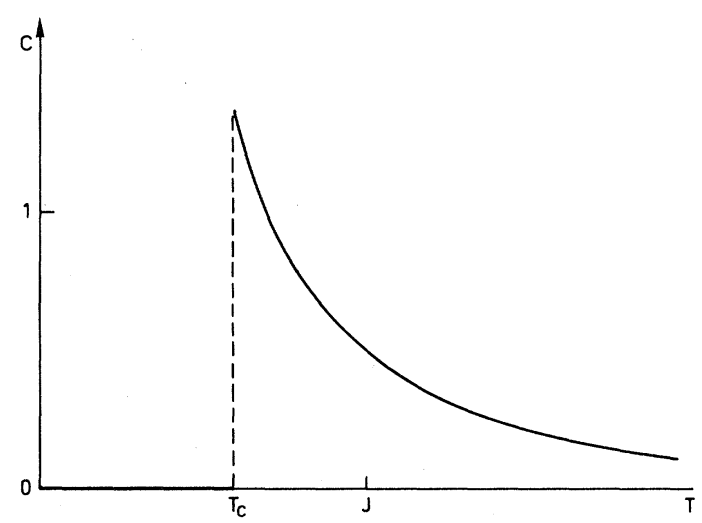

FIG. 2. The specific heat of the random-energy model as a function of temperature. 
The ground-state energy depends on the choice of the $2^{N}$ energy levels

$$
E_{\mathrm{GS}}=\min _{i}\left(E_{1}, E_{2}, \ldots, E_{2^{N}}\right)
$$

By calculations similar to those given in the Appen$\operatorname{dix} B$, one can find the fluctuations of this groundstate energy

$$
\left\langle E_{\mathrm{GS}}^{2}\right\rangle-\left\langle E_{\mathrm{GS}}\right\rangle^{2}=\frac{\pi^{2} J^{2}}{24 \ln 2}+O(1) .
$$

These fluctuations of the ground-state energy of the whole system are of order unity and so are rather small. For the random-energy model, the difficulty of finding the ground-state energy by studying finite samples comes more from the slow convergence of the average than from the fluctuations of the ground-state energy.

In the high-temperature phase, the convergence to the thermodynamic limit is more rapid because the corrections decrease exponentially with $N$. One can notice that at all the temperatures $T_{n}^{\prime}\left(T_{n}^{\prime}\right.$ $=\sqrt{2 n+1} T_{c}, n=1,2, \ldots$, ) these corrections are singular. These temperatures are probably the temperatures where the number of terms that one can use in the asymptotic expansion (26) to calculate $\langle\ln Z\rangle$ changes.

\section{REPLICA METHOD}

The replica method consists in calculating $\langle\ln Z\rangle$ by the formula

$$
\langle\ln Z\rangle=\lim _{n \rightarrow 0} \frac{\left\langle Z^{n}\right\rangle-1}{n} .
$$

We have seen in Sec. IV [Eq. (22)] that $\left\langle Z^{n}\right\rangle$ could be written as

$$
\begin{aligned}
\left\langle Z^{n}\right\rangle \sim \sum_{\langle\nu\rangle} & \frac{n !}{\prod_{p=1}^{n}\left(v_{p}\right) ! \prod_{p=1}^{n}(p !)^{\nu p}} \\
& \times \exp \left[N \sum_{p=1}^{n} v_{p}\left(\ln 2+p^{2} \frac{\lambda^{2}}{4}\right)\right] .
\end{aligned}
$$

At high temperature, the dominant term in the sum was $\nu_{1}=n$ and $\nu_{p}=0$ for $2 \leqslant p \leqslant n$. This leads to the expression (24) for $\left\langle Z^{n}\right\rangle$. Then by using Eq. (35) one finds

$$
\langle\ln Z\rangle=N\left(\ln 2+\frac{J^{2}}{4 T^{2}}\right),
$$

which is the good expression in the high-temperature phase. At low temperature, the dominant term corresponds to $\nu_{n}=1$ and $\nu_{p}=0$ for $1 \leqslant p \leqslant n-1$. One finds that $\left\langle Z^{n}\right\rangle$ is given by Eq. (25)

$$
\left\langle Z^{n}\right\rangle \sim \exp N\left(\ln 2+n^{2} \frac{J^{2}}{4 T^{2}}\right) .
$$

It is then impossible to use Eq. (35) because the $n \rightarrow 0$ limit of $\left\langle Z^{n}\right\rangle$ is not 1 .

Surprisingly it is possible to recover the lowtemperature expression of $\langle\ln Z\rangle$ by looking at another extremum in the sum (22) which is in fact a minimum. The sum (22) has to be done over all the choices of integers $v$ such that

$$
\sum_{p=1}^{n} p v_{p}=n
$$

If one forgets the fact that the $p$ and the $\nu_{p}$ are integer one can find using a Lagrange parameter that any extremum corresponds to all the $\nu_{p}=0$ except one.

This nonzero $v_{p}$ has to be equal to $n / p$. We can now find the value of $p$ for which $(n / p)(\ln 2$

$\left.+p^{2} \lambda^{2} / 4\right)$ is extremum. The result is

$$
p=\frac{2 \sqrt{\ln 2}}{\lambda}=\frac{T}{T_{c}}, \quad \nu_{p}=n \frac{T_{c}}{T} .
$$

The corresponding contribution in sum (22) is

$$
\exp N n \frac{J}{T} \cdot \sqrt{\ln 2} \text {. }
$$

So by applying the replica formula (35) to the expression (37), one recovers the true expression (17) of $\langle\ln Z\rangle$ below $T_{c}$.

This calculation needs two comments: First, the term (37) is not present in the sum (22) below $T_{c}$. In Eq. (22) the $p$ and the $\nu_{p}$ are integers verifying $1 \leqslant p \leqslant n$ and $0 \leqslant \nu_{p} \leqslant n$ and obviously the choice [Eq. (36)] does not satisfy these conditions. Secondly, even if Eq. (37) were present in Eq. (22), it would be negligible compared with the dominant contribution found in Eq. (25). So for this RE model, a blind calculation where all the constraints are forgotten can lead to the good expression of $\langle\ln Z\rangle$. It would be interesting to justify this calculation and to find an argument which shows that one has to take the $n \rightarrow 0$ limit of Eq. (37) below $T_{c}$.

The replica method can also be applied in the microcanonical ensemble. It becomes

$$
\langle S(E)\rangle=\lim _{q \rightarrow 0} \frac{\left\langle[n(E)]^{q}\right\rangle-1}{q}
$$

where $\langle S(E)\rangle$ is the average entropy at energy $E$ and $n(E)$ is the density defined in Sec. III. In Eqs. (29) and (30) we have found the behavior of all the moments $\left\langle[n(E)]^{q}\right\rangle$.

If $|E|>E_{0}$, then $\langle n(E)\rangle$ is very small compared with 1 and one has $\left\langle[n(E)]^{q}\right\rangle \sim\langle n(E)\rangle$. By apply- 
ing Eq. (38), one finds

$$
\langle S(E)\rangle=\lim _{q \rightarrow 0} \frac{\langle n(E)\rangle-1}{q}=-\infty
$$

so, we find again that there is no level at these energies.

If $|E|<E_{0}$, then $\left\langle[n(E)]^{q}\right\rangle \sim\langle n(E)\rangle^{q}$ and Eq. (38) gives

$$
\langle S(E)\rangle=\lim _{q \rightarrow 0} \frac{\langle n(E)\rangle^{q}-1}{q}=\ln [\langle n(E)\rangle],
$$

which was also established in Sec. III.

We can conclude that although the replica method was rather unsatisfactory for the partition function, it works very well here in the microcanonical ensemble.

\section{EFFECT OF A UNIFORM MAGNETIC FIELD}

The definition of the random-energy model given at the beginning of Sec. III is not sufficient to describe its magnetic properties. To define the model in a uniform magnetic field, one has to come back to the Hamiltonians $\mathcal{K}_{p}$ of Sec. II. In presence of a field $H$, these Hamiltonians become

$$
\begin{array}{ll}
\mathfrak{H}_{p}^{\prime}=\mathcal{K}_{p}-H \sum_{i=1}^{N} \sigma_{i} . & \begin{array}{l}
\text { of }\langle n(E)\rangle \text { can be obtaine } \\
\text { inant term in the sum }(41)
\end{array} \\
\frac{1}{N} \ln \langle n(E)\rangle=\max _{-1 \leqslant m \leqslant 1}\left[-\left(\frac{1+m}{2}\right) \ln \left(\frac{1+m}{2}\right)-\left(\frac{1-m}{2}\right) \ln \left(\frac{1-m}{2}\right)-(\epsilon+m h)^{2}\right],
\end{array}
$$

One can calculate again the probability distributions $P(E)$ and $P\left(E_{1}, E_{2}\right)$. As before, $P\left(E_{1}, E_{2}\right)$ is factorized and the energy becomes independent when $p \rightarrow \infty$. The difference is that now, $P(E)$ depends on the magnetization $M$ of the configuration of spins

$P(E)=\left(N \pi J^{2}\right)^{-1 / 2} \exp \left[-(E+M H)^{2} / N J^{2}\right]$.

So the random-energy model is a system of $2^{N}$ independent random-energy levels among which $\left(\begin{array}{c}N \\ (N+M) / 2)\end{array}\right)$ have a magnetization $M$ and are distributed according to Eq. (40).

Like in Sec. III, we can calculate the average of the level density $n(E)$ :

$$
\begin{aligned}
\langle n(E)\rangle=\sum_{M=-N}^{+N} & \left(\begin{array}{c}
N \\
(N+M) / 2
\end{array}\right) \frac{1}{\left(\pi N J^{2}\right)^{1 / 2}} \\
& \quad \times \exp \left(-\frac{(E+M H)^{2}}{N J^{2}}\right) d E,
\end{aligned}
$$

where the sum is done over all the possible magnetizations of the system: $M=-N+2 p$ with $0 \leqslant p \leqslant N$.

In the thermodynamic limit $N \rightarrow \infty$, the behavior of $\langle n(E)\rangle$ can be obtained by looking for the dominant term in the sum (41). One finds where $\epsilon=E / N J, m=M / N$, and $h=H / J$.

The value of $m$ which gives the maximum is the solution of

$$
m=-\tanh [2 h(\epsilon+m h)] .
$$

Because of the statistical independence of the energy levels, we can use again the argument of Sec. III.

If $(1 / N) \ln \langle n(E)\rangle$ is positive, the average number of levels at energy $E$ is very large. The fluctuations of $n(E)$ are small compared with $\langle n(E)\rangle$ and then the average entropy is

$$
S(E)=\ln \langle n(E)\rangle .
$$

If $(1 / N) \ln \langle n(E)\rangle$ is negative, the average $\langle n(E)\rangle$ is much smaller than 1 . So with probability 1 , there is no level at this energy.

So in a uniform magnetic field $H$, there is a critical energy $E_{0}(H)$. If $|E|<E_{0}(H)$, the entropy is positive and given by Eq. (44) whereas there is no energy level at energies $E$ larger than $E_{0}(H)$ in absolute value. Using the relation $(1 / T)=d S / d E$, one can find the temperature dependence of the physical quantities.

In the high-temperature phase $T>T_{c}(H)$ the magnetization, the energy and the entropy are given by

$$
\begin{aligned}
& m=\tanh \frac{H}{T}, \\
& \frac{E}{N}=-m H-\frac{J^{2}}{2 T}, \\
& \frac{S}{N}=\ln 2-\frac{m}{2} \ln \left(\frac{1+m}{1-m}\right)-\frac{1}{2} \ln \left(1-m^{2}\right)-\frac{J^{2}}{4 T^{2}},
\end{aligned}
$$

and the critical temperature $T_{c}(H)$ is the temperature where the entropy vanishes (see Fig. 3).

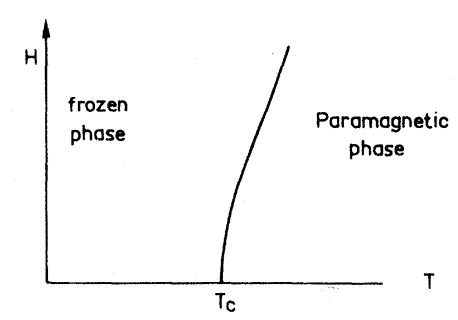

FIG. 3. The phase diagram of the random-energy model in a uniform magnetic field. 


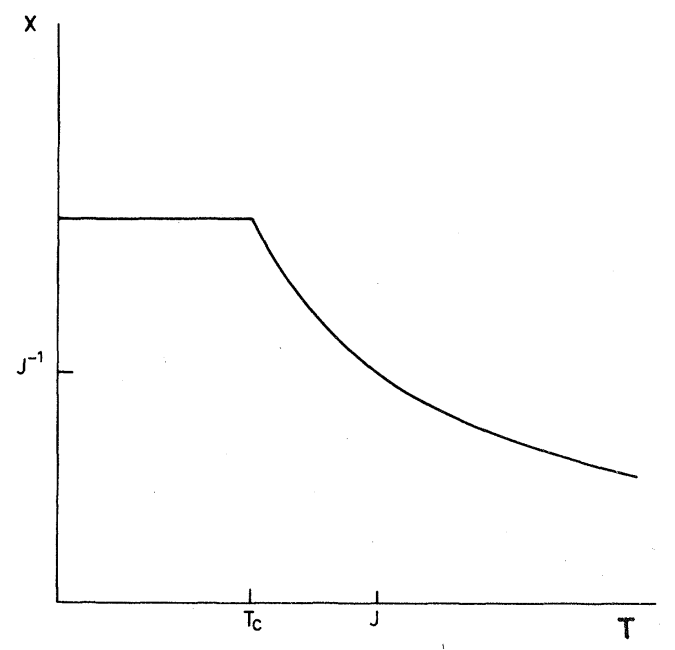

FIG. 4. The magnetic susceptibility in zero magnetic field of the random-energy model.

Below $T_{c}(H)$, the system is completely frozen in its ground state: for $T<T_{c}(H)$

$$
\begin{aligned}
& m=\tanh \left(\frac{H}{T_{c}(H)}\right), \\
& \frac{E}{N}=-m H-\frac{J^{2}}{2 T_{c}(H)}, \\
& \frac{S}{N}=0 .
\end{aligned}
$$

This gives for the magnetic susceptibility:

$$
\chi= \begin{cases}\frac{1}{T}, \text { for } T>T_{c}(H) \\ \frac{1}{T_{c}(H)}, \quad \text { for } T<T_{c}(H) .\end{cases}
$$

The susceptibility is constant (Fig. 4) in the whole low-temperature phase. A similar result was also predicted by Parisi ${ }^{12}$ for the SK model. We can also notice that the magnetization (46) in the lowtemperature phase depends only on the magnetic field and not on the temperature. A similar behavior was also proposed as a hypothesis for the SK model ${ }^{13}$ and seems to be a good approximation. ${ }^{14}$ The important point is that in spite of its simplicity, the RE model gives for the magnetic susceptibility a cusp at the transition and a constant susceptibility at low temperature.

\section{EFFECT OF FERROMAGNETIC PAIR INTERACTIONS}

It is also interesting to study the case where in addition to the random interactions, there are ferromag- netic pair interactions $J_{0}$. The Hamiltonians $\mathcal{K}_{p}$ become

$$
\mathfrak{K}_{p}^{\prime \prime}=\mathcal{K}_{p}-\frac{J_{0}}{N} \sum_{\langle i j\rangle} \sigma_{i} \sigma_{j}
$$

Like in the SK model, we have here an interaction $J_{0} / N$ for any pair of spins in the system. The presence of $N^{-1}$ in the interaction is necessary to ensure an extensive free energy. Here again in the limit $p \rightarrow \infty$, the energy levels become independent random variables. The RE model becomes a system of $2^{N}$ energy levels among which $\left(\begin{array}{c}N \\ (N+M) / 2\end{array}\right)$ have a magnetization $M$ and are distributed according to

$$
P(E) \sim \exp \left[-\left(E+\frac{M^{2} J_{0}}{2 N}\right)^{2} / N J^{2}\right] .
$$

To obtain the temperature dependence of the free energy $F$ and the magnetization $m$, the procedure is exactly the same as in Sec. VII. The results are summarized (in Fig 5).

One finds four phases: (i) in the paramagnetic phase

$$
\begin{aligned}
& m=0 \\
& F / N=-T \ln 2-\frac{J^{2}}{4 T}
\end{aligned}
$$

(ii) in the ferromagnetic phase

$$
\begin{aligned}
& m=\tanh \left(\frac{J_{0} m}{T}\right) \\
& F / N=-T \ln 2-\frac{J^{2}}{4 T}+\frac{J_{0} m^{2}}{2}+\frac{T}{2} \ln \left(1-m^{2}\right) ;
\end{aligned}
$$

(iii) in the frozen phase I

$$
\begin{aligned}
& m=0 \\
& F / N=-J \sqrt{\ln 2} ;
\end{aligned}
$$

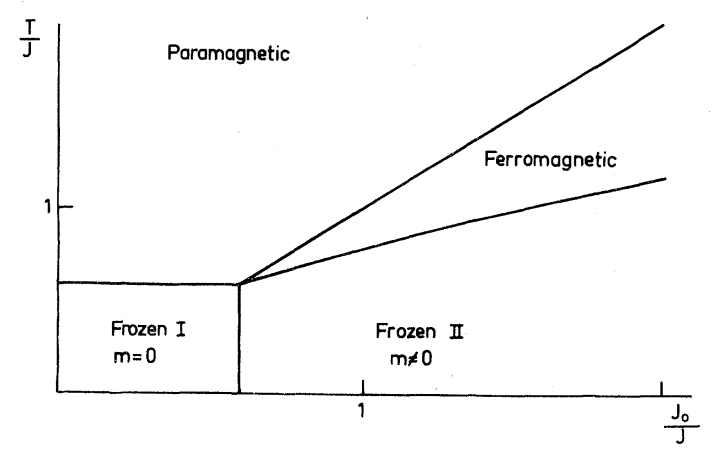

FIG. 5. The phase diagram of the random-energy model in the presence of ferromagnetic pair interactions. At low temperature, the magnetization $m$ in the frozen phase is zero if $J_{0}<J / 2 \sqrt{\ln 2}$ and is a function of $J_{0}$ if $J_{0}>J / 2 \sqrt{\ln 2}$. 
and (iv) in the frozen phase II the system is completely frozen

$$
\begin{aligned}
& m=m\left(T_{c}\left(J_{0}\right)\right) \\
& F / N=F\left(T_{c}\left(J_{0}\right)\right) / N,
\end{aligned}
$$

where $T_{c}\left(J_{0}\right)$ is the transition temperature from the ferromagnetic phase to the frozen phase II.

The lines of transition are between the paramagnetic phase and the frozen phase I $T_{c}=J / 2 \sqrt{\ln 2}$; between the paramagnetic phase and the ferromagnetic phase $T_{c}=J_{0}$; between the ferromagnetic phase and the frozen phase II. The transition temperature $T_{c}\left(J_{0}\right)$ is the temperature where the entropy of the ferromagnetic phase vanishes:

$$
\begin{aligned}
\frac{1}{N} S\left(T_{c}\left(J_{0}\right)\right)= & \ln 2-\frac{J_{0} m^{2}}{T_{c}\left(J_{0}\right)}-\frac{1}{2} \ln \left(1-m^{2}\right) \\
& -\frac{J^{2}}{4 T_{c}^{2}\left(J_{0}\right)}=0,
\end{aligned}
$$

where $m$ is solution of

$$
m=\tanh \left(\frac{J_{0} m}{T_{c}\left(J_{0}\right)}\right)
$$

between the frozen phase I and the frozen phase II

$$
J_{0}=J /(2 \sqrt{\ln 2}) \text {. }
$$

The phase diagram found here is qualitatively the same as the one predicted for the SK model. ${ }^{1}$ The properties of the RE model in presence of ferromagnetic pair interactions are closely related to its properties in a uniform magnetic field. ${ }^{15}$ So all the results of this section could have been obtained directly from those of Sec. VII.

\section{INDEPENDENT ENERGY APPROXIMATION}

The main idea of the present paper is that when the average level density $\langle n(E)\rangle$ is much smaller than 1 , then, with probability 1 , there is no level at this energy. This remains true for any spin-glass model. In this section, we are going to show that this argument provides a lower bound for the ground-state energy of some spin-glass models.

Consider now a more usual spin-glass model

$$
\mathfrak{K}=-\sum_{\langle j\rangle} J_{i j} \sigma_{i} \sigma_{j},
$$

where the spin $\sigma_{i}$ are Ising spins on a lattice and the $J_{i j}$ are random nearest-neighbor interactions distributed according to a probability distribution $\rho\left(J_{i j}\right)$. When this distribution $\rho$ is symmetric

$$
\rho\left(J_{i j}\right)=\rho\left(-J_{i j}\right)
$$

it is easy to calculate the average level density

$$
\begin{aligned}
\langle n(E)\rangle= & 2^{N} \int \ldots \int \prod_{\langle i j\rangle} \rho\left(J_{i j}\right) d J_{i j} \\
& \times \delta\left(E-\sum_{\langle i j\rangle} J_{i j}\right) d E .
\end{aligned}
$$

In Eq. (52), we have used the fact that

$$
\rho\left(J_{i j}\right)=\rho\left(J_{i j} \sigma_{i} \sigma_{j}\right) .
$$

If $E_{0}$ is the lowest energy where $N^{-1} \ln \langle n(E)\rangle$ vanishes $\left[N^{-1} \ln \langle n(E)\rangle\right.$ is negative if $\left.|E|>-E_{0}\right]$, one knows that with probability 1 , there is no level of energy $E$ smaller than $E_{0}$. This implies that $E_{0}$ is a lower bound for the ground-state energy $E_{\mathrm{Gs}}$. Let us look at two examples: the symmetric $\pm J$ model and the symmetric Gaussian model.

For the symmetric $\pm J$ model, the density $\rho\left(J_{i j}\right)$ is

$$
\rho\left(J_{i j}\right)=\frac{1}{2}\left[\delta\left(J_{i j}-J\right)+\delta\left(J_{i j}+J\right)\right] .
$$

In the thermodynamic limit, one finds

$$
\begin{aligned}
N^{-1} \ln \langle n(E)\rangle \sim & \left\{\ln 2+d \ln d-\frac{d}{2} \ln \left[d^{2}-\mid \frac{E}{N J}\right)^{2}\right\} \\
& \left.-\frac{E}{2 N J} \ln \left(\frac{d N J+E}{d N J-E}\right)\right\}
\end{aligned}
$$

where the lattice is a $d$-dimensional cubic lattice. In dimension $2^{16-18}$ the ground-state energy has been calculated by numerical studies $E_{\mathrm{GS}} / N J=-1.40$ \pm 0.01 . The value of $E_{0}$ calculated from Eq. (54) is $E_{0} / N J=-1.560$. In dimension 3, Kirkpatrick ${ }^{17}$ estimated $E_{\mathrm{GS}} / N J=-1.75$ whereas Eq. (54) gives $E_{0} / N J=-1.956$. In the high-dimension limit $d \rightarrow \infty$, one can also find the analytic expansion of $E_{0}$ :

$$
E_{0}=-N J \sqrt{2 d \ln 2}\left(1-\frac{\ln 2}{6 d}+\cdots\right) \text {. }
$$

For the symmetric Gaussian model, the distribution $\rho\left(J_{i j}\right)$ is by definition

$$
\rho\left(J_{i j}\right)=\left(2 \pi J^{2}\right)^{1 / 2} \exp \left[-\left(\frac{J_{i j}^{2}}{2 J^{2}}\right)\right] .
$$

The expression of $N^{-1} \ln \langle n(E)\rangle$ is very simple:

$$
N^{-1} \ln \langle n(E)\rangle \sim\left(\ln 2-\frac{E^{2}}{2 d N^{2} J^{2}}\right) .
$$

Here again the spins are on a $d$-dimensional cubic lattice, one finds for $E_{0}$ :

$$
E_{0} / N J=-\sqrt{2 d \ln 2} .
$$

In dimension $2, E_{0} / N J=-1.665$ whereas the result of numerical studies ${ }^{18}$ for the ground-state energy is $E_{\mathrm{GS}} / N J=-1.31 \pm 0.01$. In infinite dimension, $E_{0}$ is 
the ground-state energy of the RE model $E_{0} / N J$ $=-\sqrt{\ln 2}=-0.8326$ and it is a lower bound for the ground-state energy of the $\mathrm{SK}$ model $E_{\mathrm{GS}} / N J=$ -0.765 . It is also a lower bound for the ground-state energy of the models defined by the Hamiltonians $\mathfrak{H}_{p}$.

So we see that for a large class of models, $E_{0}$ is very easy to calculate. $E_{0}$ provides a lower bound of the ground-state energy $E_{\mathrm{GS}}$ and is a rather good approximation of $E_{\mathrm{GS}}$. For the RE model, it was possible to say that $n(E) \sim\langle n(E)\rangle$ when $\langle n(E)\rangle$ is large. This was justified by the statistical independence of the energy levels. This cannot be generalized to other spin-glass models. Because of the correlations between the energy levels, it is always possible that, when $\langle n(E)\rangle$ is large, the probability that $n(E)$ is not zero vanishes exponentially with $N$. For example, if $n(E)$ could only take two values:

$$
n(E)=\left\{\begin{array}{l}
0, \text { with probability } 1-e^{-b N} \\
e^{a N}, \text { with probability } e^{-b N}
\end{array}\right.
$$

then $\langle n(E)\rangle=e^{(a-b) N}$ which can be very large. However in this example $n(E)=0$ with probability 1 in the thermodynamic limit. This is the reason why $E_{0}$ is only a lower bound of $E_{\mathrm{Gs}}$.

\section{ATTEMPTS OF EXPANSION AROUND THE RE MODEL}

At the end of this paper, the open question is to find how to take into account the correlations between the energy levels in realistic spin-glass models. The simplest approach is to study the Hamiltonians $\mathcal{K}_{p}$ defined by Eqs. (3) and (4) because these correlations are known, and to try a large $p$ expansion: I could not find how to expand for large $p$ the quantities of interest like the average free energy $\langle\ln Z\rangle$ or the average entropy $\langle\ln n(E)\rangle$. I could only expand the moments $\left\langle Z^{n}\right\rangle$ which are of course less interesting quantities. For the Hamiltonians $\mathfrak{K}_{p}$, the calculations of the moments $\left\langle Z^{n}\right\rangle$ can be done using replicas. After the average, one finds

$$
\begin{aligned}
\left\langle Z^{n}\right\rangle=\sum_{\left\{\sigma_{i}^{\alpha}\right\}} \exp \left[\frac{J^{2}}{4 T^{2}} \frac{p !}{N^{p-1}} \underset{\left\langle i_{1}, \ldots, i_{p}\right\rangle}{\sum}\right. \\
\left.\times\left(\sum_{\alpha=1}^{n} \sigma_{i_{1}}^{\alpha} \cdots \sigma_{i_{p}}^{\alpha}\right)^{2}\right] .
\end{aligned}
$$

Equation (57) shows that the moments $\left\langle Z^{n}\right\rangle$ are very similar to the partition function of a lattice gauge theory. ${ }^{19}$ There is a local symmetry in the effective Hamiltonian $H$ :

$$
H=-\frac{J^{2}}{4 T^{2}} \frac{p !}{N^{p-1}} \sum_{\left\langle i_{1}, \ldots, i_{p}\right\rangle}\left(\sum_{\alpha=1}^{n} \sigma_{i_{1}}^{\alpha} \cdots \sigma_{i_{p}}^{\alpha}\right)^{2} .
$$

If one changes the sign of all the spins of site $i, H$ remains unchanged: $\left\{\sigma_{i}^{\alpha}\right\} \rightarrow\left\{\epsilon_{i} \sigma_{i}^{\alpha}\right\}$ with $\epsilon_{i}= \pm 1$ for any $i$. When $n$ is even, there is another gauge symmetry $\left\{\sigma_{i}^{\alpha}\right\} \rightarrow\left\{\epsilon_{\alpha} \sigma_{i}^{\alpha}\right\}$ with $\epsilon_{\alpha}= \pm 1$ for any $\alpha$. One can make a low-temperature expansion of Eq. (57) for any $p$ :

$$
\begin{aligned}
\ln \left\langle Z^{n}\right\rangle-N\left[\ln 2+n^{2} \frac{J^{2}}{4 T^{2}}\right. & \\
& \left.+g(n) \exp \left(-\frac{J^{2}}{T^{2}}(n-1) p\right)+\cdots\right],
\end{aligned}
$$

where

$$
g(n)= \begin{cases}n, & \text { for } n \geqslant 3 \\ 1, & \text { for } n=2 \\ 0, & \text { for } n=1\end{cases}
$$

In the expansion (58), it is clear that all the Hamiltonians $\mathfrak{K}_{p}$ give the same behavior of the moments $\left\langle Z^{n}\right\rangle$ at very low temperature. As the continuation of Eq. (25) could not provide $\langle\ln Z\rangle$, it seems difficult to extract the first correction of the free energy from Eq. (58). There is some hope to calculate also the moments $\left\langle[n(E)]^{q}\right\rangle$ for the Hamiltonians $\mathcal{H}_{p}$ but this requires more complicated calculations.

\section{CONCLUSION}

The random-energy model is obviously an extreme simplification of spin-glass problems. Its properties are certainly different from those of real materials and it is hard to expect more than qualitative agreement with experimental data. From a theoretical point of view, it has the advantage to be exactly solvable. It shows the limitations of the replica method. The freezing of the system below its transition can be well understood by looking at the energy-level density. The phase diagrams of the RE model are qualitatively the same as in the SK model. The magnetic susceptibility is constant in the whole low-temperature phase. So the simplification of considering independent random energies has not suppressed the physical interest of the model.

The RE model provides also lower bounds for the ground-state energies of a large class of spin-glass models. It would be interesting to go further than the independent energy approximation by including the correlations between the energy levels. To do so, the simplest models seem to be the Hamiltonians $\mathcal{H}_{p}$ in the limit $p \rightarrow \infty$. It would also be interesting to understand the reason why a blind calculation by the replica method leads to the true free energy. Maybe this calculation has connections with the broken sym- 
metry of replicas introduced by $\mathrm{Parisi}^{7}$ for the SK model.

\section{ACKNOWLEDGMENTS}

I wish to thank the Group de Physique des Solides of the Ecole Normale Supérieure and the Service de Physique Théorique of the Centre d'Etudes Nucléaires Saclay, especially P. Moussa, Y. Pomeau, G. Toulouse, and J. Vannimenus for many stimulating discussions.

\section{APPENDIX A}

The question of calculating the average free energy $\langle\ln Z\rangle$ from the expression of the moments $\left\langle Z^{n}\right\rangle$ is not exactly a problem of analytic continuation. The fact that the $\left\langle Z^{n}\right\rangle$ are the moments of a distribution and not only numbers imposes constraints on the continuation to noninteger values of $n$.

In this appendix, I give an example which illustrates that when the moments $\left\langle x^{n}\right\rangle$ increase too rapidly, the measure is not determined uniquely. Note that the growth of the moments $\left(\exp \lambda n^{2}\right)$ is the same as for the moments $\left\langle Z^{n}\right\rangle$ of the partition function of the RE model. Consider the family of measures $d \mu(x)$ defined by

$$
d \mu(x)=\frac{\sum_{n=-\infty}^{+\infty} b^{-n^{2} / 2-n \nu} \delta\left(x-b^{n+v}\right)}{\sum_{n=-\infty}^{+\infty} b^{-n^{2} / 2-n \nu}} d x
$$

These measures depend on two parameters ( $b$ and $v)$. Each value of $v\left(-\frac{1}{2}<v \leqslant \frac{1}{2}\right)$ defines a new measure. It is easy to calculate the moments $\left\langle x^{n}\right\rangle$. One finds that for any integer $n$ the moments $\left\langle x^{n}\right\rangle$ do not depend on $\nu$ :

$$
\left\langle x^{n}\right\rangle=b^{n^{2} / 2} \text {. }
$$

One can also calculate $\langle\ln x\rangle$

$$
\langle\ln x\rangle \sim \nu \ln b, \text { for large } b .
$$

So two different measures corresponding to different values of $v$ give different answers for $\langle\ln x\rangle$ even if all their moments are exactly the same.

\section{APPENDIX B}

In this appendix, we obtain the free energy of the random-energy model.

In order to calculate the average of $\ln Z$, one can use an integral representation of the logarithm:

$$
\langle\ln Z\rangle=\int_{0}^{\infty} \frac{e^{-t}-\left\langle e^{-t Z}\right\rangle}{t} d t
$$

After a partial integration, one finds

$$
\langle\ln Z\rangle=\int_{0}^{\infty} \ln t e^{-t} d t-\int_{0}^{\infty} \ln t e^{-\phi} d \phi,
$$

where $\phi$ is defined by

$$
e^{-\phi}=\left\langle e^{-t Z}\right\rangle
$$

The only question, now, is to find an explicit expression of $t$ as a function of $\phi$, where $t$ and $\phi$ are related by Eq. (B3).

As the partition function is

$$
Z=\sum_{i=1}^{2^{N}} e^{-\beta E_{i}}
$$

and the energy levels are random variables with probability distribution

$$
p\left(E_{i}\right)=\frac{1}{\left(\pi N J^{2}\right)^{1 / 2}} \exp \left(-\frac{E_{i}^{2}}{N J^{2}}\right)
$$

one has

$$
\left.e^{-\phi}=\left[\frac{1}{\left(\pi N J^{2}\right)^{1 / 2}} \int_{-\infty}^{+\infty} d E \exp \left(-\frac{E^{2}}{N J^{2}}-t e^{-\beta E}\right)\right]\right]^{2^{N}}
$$

or

$\exp \left(-\frac{\phi}{2^{N}}\right)=\frac{1}{\sqrt{\pi}} \int_{-\infty}^{+\infty} d y \exp \left(-y^{2}-t e^{-\lambda y}\right)$,

with

$$
\lambda=\sqrt{N} \beta J,
$$

Notice that the definition of $\lambda$ in this appendix is not the same as in the body of the paper.

We are now going to obtain the asymptotic behavior (when $\lambda$ is large) of the integral in the right-hand side of Eq. (B5).

If we call

$$
f(t)=\frac{1}{\sqrt{\pi}} \int_{-\infty}^{+\infty} d y \exp \left(-y^{2}-t e^{-\lambda y}\right)
$$

we shall prove that: if $-p \lambda^{2} / 2<\ln t<-(p-1) \lambda^{2} / 2$, where $p$ is an integer then

$$
f(t)=1-t e^{\lambda^{2} / 4}+\frac{t^{2}}{2 !} e^{\lambda^{2}}+\cdots+(-)^{p-1} \frac{t^{p-1}}{(p-1) !} e^{(p-1)^{2} \lambda^{2} / 4}+\frac{1}{\sqrt{\pi} \lambda} e^{-\ln ^{2} t / \lambda^{2}}\left[\Gamma\left(\frac{2 \ln t}{\lambda^{2}}\right)-\frac{1}{\lambda^{2}} \Gamma^{\prime \prime}\left(\frac{2 \ln t}{\lambda^{2}}\right)+O\left(\frac{1}{\lambda^{4}}\right)\right]
$$


Proof:

In Eq. (B7), we can make the change of variable:

$$
t e^{-\lambda y}=u \text {. }
$$

It follows that:

$$
f(t)=\frac{1}{\sqrt{\pi} \lambda} \exp \left(-\frac{\ln ^{2} t}{\lambda^{2}}\right) \int_{0}^{\infty} \exp \left[\left(\frac{2 \ln t}{\lambda^{2}}-1\right) \ln u-\frac{\ln ^{2} u}{\lambda^{2}}-u\right] d u
$$

The convergence of the integral is ensured by the presence of the term $-\ln ^{2} u$ in the exponential. Nevertheless, when $\ln t$ is positive, this term is not necessary and one can expand $\exp \left(-\ln ^{2} u / \lambda^{2}\right)$ and obtain: If $\ln t>0$ :

$$
f(t)=\frac{1}{\sqrt{\pi} \lambda} e^{-\ln ^{2} t / \lambda^{2}}\left[\Gamma\left(\frac{2 \ln t}{\lambda^{2}}\right)-\frac{1}{\lambda^{2}} \Gamma^{\prime \prime}\left(\frac{2 \ln t}{\lambda^{2}}\right)+\cdots+\left(-\frac{1}{\lambda^{2}}\right)^{p} \frac{1}{p !} \Gamma^{(2 p)}\left(\frac{2 \ln t}{\lambda^{2}}\right)+O\left(\frac{1}{\lambda^{2 p+2}}\right)\right]
$$

This proves the formula (B8) when $p=0$.

Let us now consider the case $p \geqslant 1$. By deriving $p$ times Eq. (B7) with respect to $t$, it is easy to prove that

$$
f^{(p)}(t)=(-)^{p} e^{p^{2} \lambda^{2} / 4} f\left(t e^{p \lambda^{2} / 2}\right)
$$

If $t$ is chosen such that: $-p \lambda^{2} / 2<\ln t<-[(p-1) / 2] \lambda^{2}$ then, $\ln \left(t e^{p \lambda^{2} / 2}\right)$ is positive and one can replace $f\left(t e^{p \lambda^{2} / 2}\right)$ in Eq. (B11) by its expansion Eq. (B10). So Eq. (B11) becomes

$$
f^{(p)}(t)=(-)^{p} e^{p^{2} \lambda^{2} / 4} \exp \left[-\ln ^{2}\left(t e^{p \lambda^{2} / 2}\right) / \lambda^{2}\right]\left[\Gamma\left(\frac{2 \ln t}{\lambda^{2}}+p\right)-\frac{1}{\lambda^{2}} \Gamma^{\prime \prime}\left(\frac{2 \ln t}{\lambda^{2}}+p\right)+O\left(\frac{1}{\lambda^{4}}\right)\right] .
$$

One can now find the general solution of the differential equation (B12).

$$
f(t)=\alpha_{0}+\alpha_{1} t+\cdots \alpha_{p-1} t^{p-1}+\frac{1}{\sqrt{\pi} \lambda} \exp \left(-\frac{\ln ^{2} t}{\lambda^{2}}\right)\left[\Gamma\left(\frac{2 \ln t}{\lambda^{2}}\right)-\frac{1}{\lambda^{2}} \Gamma^{\prime \prime}\left(\frac{2 \ln t}{\lambda^{2}}\right)+O\left(\frac{1}{\lambda^{4}}\right)\right),
$$

where $\alpha_{0}, \alpha_{1}, \ldots, \alpha_{p-1}$ are a priori the unknown constants of integration of the differential equation.

We can determine these constants using the Taylor expansion of $f(t)$ around $t=0$. From Eqs. (B7) and (B11), one has

$$
f^{(p)}(0)=(-)^{p} e^{p^{2} \lambda^{2} / 4}
$$

So the Taylor expansion of $f(t)$ is

$$
f(t)=1-t e^{\lambda^{2} / 4}+\cdots+(-)^{p-1} \frac{t^{p-1}}{(p-1) !} e^{(p-1)^{2} \lambda^{2} / 4}+R_{p}(t)
$$

where $R_{p}(t)=\left(t^{p} / p !\right) f^{(p)}(\theta t)$ with $0 \leqslant \theta \leqslant 1$. One can easily find a majoration for this rest using the fact that

$$
\left|f^{(p)}(\theta t)\right| \leqslant\left|f^{(p)}(0)\right|=e^{\lambda^{2} p^{2} / 4} .
$$

In Eq. (B14), the rest is smaller than any term of the sum when $t<e^{-p \lambda^{2} / 2+\lambda^{2} / 4}$. This means that if $\ln t / \lambda^{2}<-p / 2+\frac{1}{4}$, then

$$
f(t)=1-e^{\lambda^{2} / 4} t+\cdots+(-)^{p-1} \frac{e^{\lambda^{2}(p-1)^{2} / 4}}{(p-1) !} t^{p-1}+O\left(e^{\lambda^{2} p^{2} / 4} t^{p}\right) .
$$

By comparing the two expansions (B13) and (B15) for $-p / 2<\ln t / \lambda^{2}<-p / 2+\frac{1}{4}$ one gets:

$$
\alpha_{i}=\frac{f^{(i)}(0)}{i !}
$$

and so the expansion (B8) is proved as the $\alpha_{i}$ are necessarily constant for $-p / 2<\ln t / \lambda^{2}<(-p+1) / 2$. 
From Eqs. (B5) and (B8), we can now write $t$ as an explicit function of $\phi$ :

$$
\begin{aligned}
& \text { If }-\frac{\lambda^{2}}{2}<\ln t<0 \\
& \exp \left(-\frac{\phi}{2^{N}}\right)=1+\Gamma\left(\frac{2 \ln t}{\lambda^{2}}\right) \frac{1}{\sqrt{\pi} \lambda} \exp \left(\frac{\ln ^{2} t}{\lambda^{2}}\right)+\cdots .
\end{aligned}
$$

Therefore one can find $\ln t$ as a function of $\phi$ for large $N$

$$
\ln t=-\frac{N J}{T} \sqrt{\ln 2}+\frac{T_{c}}{T}\left\{\frac{1}{2} \ln N+\ln \phi-\ln \left[-\Gamma\left(-\frac{T}{T_{c}}\right)\right]+\frac{1}{2} \ln \pi-\ln \left(\frac{T}{J}\right)\right\}+O(1)
$$

where

$$
T_{c}=\frac{J}{2 \sqrt{\ln 2}}
$$

Using Eq. (B17), one can verify that condition (B16) can be written $T<T_{c}$ and from Eq. (B2), one gets

$$
\langle\ln Z\rangle=\frac{N J}{T} \sqrt{\ln 2}-\frac{T_{c}}{2 T} \ln N+\left(1-\frac{T_{c}}{T}\right) \Gamma^{\prime}(1)-\frac{1}{2} \frac{T_{c}}{T} \ln (4 \pi \ln 2)+\frac{T_{c}}{T} \ln \left[\Gamma\left(1-\frac{T}{T_{c}}\right)\right]+O(1) .
$$

If

$$
\begin{aligned}
& -p \lambda^{2} / 2<\ln t<-(p-1) \lambda^{2} / 2 \text { with } p \geqslant 2 \\
& \exp \left(-\frac{\phi}{2^{N}}\right)=1-t e^{\lambda^{2} / 4}+\frac{t^{2}}{2 !} e^{\lambda^{2}}+\cdots+(-)^{p-1} \frac{t^{p-1}}{(p-1) !} e^{(p-1)^{2} \lambda^{2} / 4}+\frac{1}{\sqrt{\pi} \lambda} \exp \left(-\frac{\ln { }^{2} t}{\lambda^{2}}\right) \Gamma\left(\frac{2 \ln t}{\lambda^{2}}\right)+\cdots
\end{aligned}
$$

We can again calculate $\ln t$ as a function of $\phi$

$$
\begin{aligned}
\ln t= & -N \ln 2-\frac{\lambda^{2}}{4}+\ln \phi+Q_{p}\left(\frac{\phi}{2^{N}}, e^{\lambda^{2} / 4}\right) \\
& +\frac{1}{\sqrt{\pi} \lambda} \exp \left[-\frac{1}{\lambda^{2}}\left(N \ln 2-\frac{\lambda^{2}}{4}\right)^{2}+\frac{2}{\lambda^{2}}\left(N \ln 2-\frac{\lambda^{2}}{4}\right) \ln \phi\right] \Gamma\left(-\frac{1}{2}-\frac{2 N \ln 2}{\lambda^{2}}\right)+\cdots,
\end{aligned}
$$

where $Q_{p}\left(\phi / 2^{N}, e^{\lambda^{2} / 4}\right)$ is a polynomial of its two variables $\phi / 2^{N}, e^{\lambda^{2} / 4}$. For example:

$$
\begin{aligned}
& Q_{2}\left(\frac{\phi}{2^{N}}, e^{\lambda^{2} / 4}\right)=0 \\
& Q_{3}\left(\frac{\phi}{2^{N}}, e^{\lambda^{2} / 4}\right)=\frac{1}{2} \frac{\phi}{2^{N}}\left(e^{\lambda^{2} / 2}-1\right) \\
& Q_{4}\left(\frac{\phi}{2^{N}}, e^{\lambda^{2} / 4}\right)=\frac{1}{2} \frac{\phi}{2^{N}}\left(e^{\lambda^{2} / 2}-1\right)-\frac{1}{24} \frac{\phi^{2}}{2^{2 N}}\left(4 e^{3 \lambda^{2} / 2}-9 e^{\lambda^{2}}+6 e^{\lambda^{2} / 2}-1\right)
\end{aligned}
$$

Using Eq. (B20), one sees that condition (B19) becomes $\sqrt{2 p-3} T_{c}<T<\sqrt{2 p-1} T_{c}$. We can now calculate $\langle\ln Z\rangle$ using Eqs. (B2) and (B6)

$$
\begin{aligned}
\langle\ln Z\rangle= & N \ln 2+\frac{N J^{2}}{4 T^{2}}-\int_{0}^{\infty} d \phi e^{-\phi} Q_{p}\left(\frac{\phi}{2^{N}}, e^{N J^{2} / 4 T^{2}}\right) \\
& +\frac{2 \sqrt{\pi} T}{J\left(\frac{T^{2}}{T_{c}^{2}}+1\right) \sqrt{N} \sin \left[\frac{\pi}{2}\left(\frac{T^{2}}{T_{c}^{2}}+1\right)\right]} \exp \left[-\frac{N T^{2} J^{2}}{16}\left(\frac{1}{T_{c}^{2}}-\frac{1}{T^{2}}\right)^{2}\right]+\cdots
\end{aligned}
$$


The term $\sin \left\{\pi / 2\left[\left(T^{2} / T_{c}^{2}\right)+1\right]\right\}$ comes from the product of two $\Gamma$ functions

$$
[\Gamma(z) \Gamma(-z)=-\pi / z \sin \pi z] .
$$

The integral of the polynomial $Q$ gives a polynomial of two variables $\left(2^{-N}\right.$ and $\left.e^{N J^{2} / 4 T^{2}}\right)$. The important fact is that this term is exponentially small in $N$ and is regular at all the temperatures $T_{n}^{\prime}=T_{c} \sqrt{2 n+1}$. On the other hand the last term given in the asymptotic expansion (B21) of $\langle\ln Z\rangle$ is singular at these temperatures even if it decreases also exponentially with $N$. For $n=1,2$ and 3, I could express Eq. (B21) as: for $\sqrt{2 n-1} T_{c}<T<\sqrt{2 n+1} T_{c}$

$$
\begin{aligned}
\langle\ln Z\rangle= & N\left(\ln 2+\frac{J^{2}}{4 T^{2}}\right)-\frac{1}{2}\left\langle\left(\frac{Z}{\langle Z\rangle}-1\right)^{2}\right\rangle+\frac{1}{3}\left\langle\left(\frac{Z}{\langle Z\rangle}-1\right)^{3}\right\rangle+\cdots+\frac{(-)^{n+1}}{n}\left\langle\left(\frac{Z}{\langle Z\rangle}-1\right)^{n}\right\rangle \\
& +\frac{2 \sqrt{\pi} T}{J\left(\frac{T^{2}}{T_{c}^{2}}+1\right) \sqrt{N} \sin \frac{\pi}{2}\left(\frac{T^{2}}{T_{c}^{2}}+1\right)} \exp \left[-\frac{N T^{2} J^{2}}{16}\left(\frac{1}{T_{c}^{2}}-\frac{1}{T^{2}}\right)^{2}\right]+\cdots,
\end{aligned}
$$

by adding to Eq. (B21) terms which are smaller than the terms neglected. This formula is probably true for any $n \geqslant 1$ but I did not succeed in proving it.

When one approaches $T_{c}$ from above, the integral of $Q$ in Eq. (B21) is zero and one sees that the first correction to the thermodynamic limit is singular at
$T_{c}$ as it is in the case of the SK model. It is rather striking that all the temperatures $T_{n}^{\prime}=\sqrt{2 n+1} T_{c}$ appear as singular temperatures for the corrections to the thermodynamic limit. Therefore hightemperature expansions for this random-energy model are expected to be very singular.
${ }^{1}$ D. Sherrington and S. Kirkpatrick, Phys. Rev. Lett. 35, 1972 (1975); Phys. Rev. B 17, 4384 (1978).

${ }^{2}$ D. J. Thouless, P. W. Anderson, and R. G. Palmer, Philos. Mag. 35, 593 (1977).

${ }^{3}$ S. F. Edwards and P. W. Anderson, J. Phys. F $\underline{5}, 965$ (1975).

${ }^{4}$ J. L. van Hemmen and R. G. Palmer, J. Phys. A $\underline{12}, 563$ (1979).

${ }^{5}$ J. R. L. de Almeida and D. J. Thouless, J. Phys. A 11,983 (1978).

${ }^{6}$ A. Blandin, M. Gabay, and T. Garel, J. Phys. C $\underline{13}, 403$ (1980).

${ }^{7}$ G. Parisi, J. Phys. A $\underline{13}, 1101$ (1980).

${ }^{8}$ B. Derrida, Phys. Rev. Lett. 45,79 (1980).

${ }^{9}$ D. Sherrington, J. Phys. A 13,637 (1980).

${ }^{10}$ N. I. Akhiezer, in The Classical Moment Problem, edited by
D. E. Rutherford (Oliver and Boyd, Edinburgh, 1965), pp. 29-89.

${ }^{11}$ R. G. Palmer and C. M. Pond, J. Phys. F 9, 1451 (1979).

${ }^{12}$ G. Parisi, J. Phys. A $\underline{13}, 1887$ (1980); Philos. Mag. B $\underline{41}$, 677 (1980).

${ }^{13}$ G. Parisi and G. Toulouse, J. Phys. (Paris) Lett. $\underline{41}$, L361 (1980).

${ }^{14} \mathrm{~J}$. Vannimenus, G. Toulouse, and G. Parisi, J. Phys. (Paris) 42, 565 (1981).

${ }^{15} \mathrm{G}$. Toulouse, J. Phys. (Paris) Lett. 41, L447 (1980).

${ }^{16} \mathrm{~J}$. Vannimenus and G. Toulouse, J. Phys. C 10 L537 (1977).

${ }^{17}$ S. Kirkpatrick, Phys. Rev. B $\underline{16}, 4630$ (1977).

${ }^{18}$ I. Morgenstern and K. Binder, Phys. Rev. B 22, 288 (1980).

${ }^{19}$ J. M. Drouffe and C. Itzykson, Phys. Rep. 38, 133 (1978). 